\title{
Niveis séricos de adipocinas como preditores no sucesso do tratamento de tabagistas
}

\begin{abstract}
RESUMO
Essa pesquisa teve como objetivo geral analisar a associação entre os níveis séricos das adipocinas leptina e adiponectina e da grelina com a fissura e abstinência em tabagistas. 0 método adotado foi uma pesquisa de abordagem quantitativa, tendo como campo o CIPIT/HU-UFJF. Foram analisadas as concentrações séricas das adipocinas adiponectina e leptina e da grelina correlacionando com o status tabágico no início de tratamento e quatro meses após. Os dados foram testados quanto à normalidade e, após, aplicados testes, usando o SPSS. Os resultados apontaram que não houve significância nos dados antropométricos, nos níveis séricos de adiponectina ajustada ao IMC e de grelina. Já os niveis séricos de leptina ajustados para circunferência de cintura tiveram significância. Quanto ao nível sérico de adiponectinas, estava elevado no início do tratamento naqueles que pararam de fumar. De acordo com o QSU houve redução do consumo em todos os participantes, caracterizando redução de danos com o tratamento. Conclui-se que as adipocinas podem ser consideradas, não somente marcadores para fissura e risco de recaída, mas também preditores para o sucesso do tratamento de tabagistas; a leptina como marcador para fissura e risco de recaída e; a adiponectina como marcador e preditor para cessação tabágica, contribuirá significativamente para o sucesso do tratamento de tabagistas.
\end{abstract}

DESCRITORES: Adipocinas; Fissura; Abstinência; Hábito de Fumar; Tabagismo.

\section{ABSTRACT}

This research aimed to analyze the association between adipokines serum levels leptin and adiponectin and ghrelin with craving and abstinence in smokers. The adopted method was a quantitative approach research, having as field the CIPIT/HU-UFJF. Serum concentrations of adipokines adiponectin and leptin and ghrelin were correlated with smoking status at the beginning of treatment and four months after. Data were tested for normality and then tested using SPSS. The results showed that there was no significance in anthropometric data, BMI-adjusted serum adiponectin and ghrelin levels. Serum leptin levels adjusted for waist circumference were significant. As for serum adiponectin's, it was elevated at the beginning of treatment in those who stopped smoking. According to the QSU there was a reduction in consumption in all participants, characterizing harm reduction with treatment. It is concluded that adipokines can be considered not only markers for craving and risk of relapse, but also predictors of successful treatment of smokers; leptin as a marker for cleft and risk of relapse and; Adiponectin as a marker and predictor of smoking cessation will significantly contribute to the successful treatment of smokers.

KEYWORDS: Adipokines; Fissure; Abstinence; Smoking Habit; Smoking.

\section{RESUMEN}

Esta investigación tuvo como objetivo analizar la asociación entre los niveles séricos de adipocinas leptina y adiponectina y grelina con el deseo y la abstinencia en los fumadores. El método adoptado fue una investigación de enfoque cuantitativo, teniendo como campo el CIPIT/HU-UFJF. Las concentraciones séricas de adipocinas, adiponectina y leptina y grelina se correlacionaron con el tabaquismo al comienzo del tratamiento y cuatro meses después. Los datos fueron probados para normalidad y luego probados usando SPSS. LoS resultados mostraron que no había significación en los datos antropométricos, los niveles de adiponectina sérica ajustados por el IMC y los niveles de grelina. Los niveles séricos de leptina ajustados por la circunferencia de la cintura fueron significativos. En cuanto a las adiponectinas séricas, se elevó al comienzo del tratamiento en aquellos que dejaron de fumar. Según la QSU, hubo una reducción en el consumo en todos los participantes, caracterizando la reducción de daños con el tratamiento. Se concluye que las adipocinas pueden considerarse no solo marcadores de deseo y riesgo de recaída, sino también predictores del tratamiento exitoso de los fumadores; leptina como marcador de hendidura y riesgo de recaída y; La adiponectina como marcador y predictor de dejar de fumar contribuirá significativamente al tratamiento exitoso de los fumadores.

PALABRAS CLAVE: Adipocinas; Fisura; Abstinencia; Hábito de Fumar; Fumar.

RECEBIDO EM: 06/11/2019 APROVADO EM: 07/11/2019 


\title{
Sonia Silva Paiva
}

Enfermeira. Doutora em Ciências Biomédicas- UFF. Professora Adjunta da FMP/FASE. Petrópolis, RJ. Brasil.

\author{
Aline Silva de Aguiar \\ Nutricionista. Doutora em Patologia- UFF. Professora Associada Il da Universidade Federal de Juiz de Fora.Juiz de Fora, MG. Brasil.

\section{Linda Paiva Gonçalves de Oliveira e Silva} \\ Acadêmica de Medicina- FMP. Petrópolis, RJ. Brasil.
}

\section{Gabriel Pessanha Amorim}

Graduando em Medicina- FMP.Petrópolis, RJ. Brasil.

\section{Alex Leonardo de Souza}

Enfermeiro. Especialista em Terapia Intensiva- FASE. Professor - FASE. Petrópolis, RJ. Brasil.

\section{Boaz Avellar}

Médico cardiologista. Mestrado em Gestão de Sistemas de Engenharia- UCP. Petrópolis, RJ. Brasil.

\section{Vilma Aparecida da Silva Fonseca}

Médica psiquiatra. Doutora em Psicofarmacologia- UNIFESP. Professora Titular da UFF. Niterói, RJ. Brasil.

\section{INTRODUÇÃO}

D ados epidemiológicos apontam que 5,1 milhões de fumantes morrem ao ano por doenças decorrentes do consumo do tabaco, sendo mundialmente uma das maiores causas de morbidade e mortalidade e torna-se ainda mais alarmante se comparados na questão de gênero, visto que $64 \%$ dos óbitos em adultos decorrentes do tabagismo são de mulheres. Estima-se, em geral, que dez milhões de pessoas morrerão ao ano até 2030 nas Américas por complicações decorrentes do tabagismo, sendo $80 \%$ nos países em desenvolvimento. Mais de um bilhão de pessoas fumam no mundo todo ${ }^{(1,2)}$.

A OMS incluiu o tabagismo na Classificação Internacional de Doenças e Problemas Relacionados à Saúde como "Dependência do Tabaco" (F 17.2), que integra o grupo dos transtornos mentais e comportamentais decorrentes do uso de substâncias psicoativas. O tabagismo é a maior causa isolada evitável de adoecimento e mortes precoces em todo o mundo ${ }^{(3)}$.

Critérios diagnósticos para o transtorno de uso do tabaco são estabelecidos no recente Manual Diagnóstico e Estatístico de Transtornos Mentais/ DSM V ${ }^{(4)}$, tais como: padrão de uso problemático, definição clínica de tolerância e de critérios de abstinência e ainda a definição de craving ou fissura.
Diante da gravidade do quadro decorrente do tabagismo, a OMS lançou em 2008 o plano de medidas para reduzir a epidemia do tabagismo. Neste, foram adotados seis componentes como diretrizes a serem implantadas, conhecidas como MPOWER (Monitor, protect, offer, warn, enforce, raise), a saber: monitorar o uso do tabaco e políticas de prevenção; proteger a população contra a fumaça do tabaco; oferecer ajuda para cessação do tabaco; advertir sobre os perigos do tabaco; fazer cumprir as proibições sobre publicidade promoção e patrocínio do tabaco e aumentar os impostos sobre o tabaco.

Nesse contexto, o Brasil tem se empenhado em cumprir as diretrizes MPOWER por meio do Programa Nacional de Controle do Tabagismo (PNCT), o qual tem como objetivo reduzir a prevalência de fumantes em nosso país e a consequente morbimortalidade por doenças relacionadas ao consumo do tabaco ${ }^{(5)}$.

Face à gravidade do tabagismo e em consonância com as diretrizes do MPOWER, no que se refere a oferecer auxílio para as pessoas deixarem o uso do tabaco, estudos baseados em evidencias científicas tornam-se fundamentais para pautarem as ações dos profissionais nos diversos níveis de atenção em saúde, na perspectiva do Sistema Único de Saúde (SUS).

O consumo de drogas, incluindo o ta- baco, reflete diretamente em transtornos decorrentes do uso e em necessidades e demandas de serviços de saúde que precisam estar preparados para o tratamento desta clientela. Considera-se tratamento como processo que começa quando o usuário de substâncias psicoativas entra em contato com serviços de saúde, devendo continuar por meio de uma sucessão de ações concretas até alcançar um nível de saúde e bem- estar elevados. Tratamento e reabilitação se definem como um enfoque integral destinado à identificação, assistência, atenção à saúde e integração social das pessoas que apresentam problemas provocados pelo uso de qualquer substância psicoativaa ${ }^{(6)}$.

Sabe-se que o consumo do tabaco, decorrente da nicotina, causa a sensação de bem-estar e prazer, em virtude de sofrerem influência dos mecanismos compensatórios. As substâncias psicoativas agem no sistema límbico interferindo na liberação de dopamina no núcleo accumbens, ativando o sistema de recompensa e $\operatorname{prazer}^{(7)}$.

Segundo estudo ${ }^{(8)}$, os mesmos neurotransmissores e hormônios que controlam o consumo alimentar e o peso corporal regulam também a sensibilidade à recompensa da droga no cérebro. Em levantamento bibliográfico nas principais bases de dados científicas, constatam-se poucos artigos relacionando o uso de tabaco aos hormônios, entretanto, um número bem 
reduzido de pesquisadores correlaciona à fissura, ou craving, e a abstinência ao mecanismo de ação das adiponectinas, leptinas ou grelinas, isoladamente à alguma específica, evidenciando a possibilidade de que algum deles possa ser marcador para a fissura em tabagistas. Craving é definido como um desejo de repetir a experiência dos efeitos de uma dada substância( ${ }^{(3)}$. Fissura é um termo popularmente usado pelos dependentes químicos, não sendo uma nomenclatura plenamente aprovada no meio acadêmico, sendo preferido por este o termo craving ${ }^{(9)}$, embora o Ministério da Saúde adote fissura em seus registros.

Para autores ${ }^{(10)}$, a leptina e o cortisol influenciam no tratamento de tabagismo, demonstrando em seus estudos que há redução da fissura após um mês de tratamento em mulheres que pararam de fumar. A leptina é sugerida, não somente como marcador para o tratamento do tabagismo, mas também para o tratamento do alcoolismo, apontando ainda este estudo que a testosterona inibe a secreção de leptina, justificando, assim, a diferença na eficácia no que se refere a gênero no tratamento para alcoolistas e tabagistas ${ }^{(11,12)}$.

Estudo científico ${ }^{(13)}$ aponta em seus achados que há diminuição dos níveis séricos de leptina entre fumantes em comparação a não fumantes. A leptina é uma molécula sinalizadora derivada de adipócitos, responsável por limitar a ingestão de alimentos e promover o aumento do gasto energético por interação com receptores específicos localizados no sistema nervoso central (SNC) e nos tecidos periféricos. São responsáveis pela regulação hipotálamo-pituitária-adrenocortical-HPA, inibindo o cortisol em resposta ao estresse.

A desregulação do sistema neuroendócrino, em particular do eixo HPA, tem sido associado com uma variedade de transtornos psiquiátricos. Dados de estudos clínicos e pré-clínicos sugerem que o comprometimento da função do eixo HPA e a regulação de cortisol em resposta ao estresse estão associados diretamente com o aumento da ingestão do álcool e da recaída ao tabaco ${ }^{(12,14)}$. Autores ${ }^{(12)}$ recomendam que deve ser realizado o acom- panhamento dos níveis séricos de leptina como sugestão de marcador biológico para a fissura e mudanças de humor em tabagistas em abstinência.

A adiponectina é uma proteína produzida pelos adipócitos, a qual a sua produção depende do estado nutricional, descoberta em 1995. Seu papel fisiológico não está totalmente esclarecido, mas já é conhecida a sua atuação favorável sobre a regulação da glicose e dos ácidos graxos, bem como o mecanismo central da fome e do gasto energético ${ }^{(15)}$. Para autores ${ }^{(16)}$, as mudanças nas adipocitocinas e os níveis de grelina podem ser associadas com as características de dependência.

A grelina é um hormônio produzido pelo estômago que quando está vazio, age no cérebro e dispara a sensação de fome. Autores $^{(17)}$ relataram nas suas investigações que há redução do nível de grelina associado ao aumento de leptina durante a abstinência do tabaco. Esta também pode aumentar os níveis de adiponectina, principalmente quando há ganho de peso. Estas alterações dos neuromoduladores do apetite durante a abstinência podem interferir na sua manutenção ${ }^{(18)}$.

Diante do exposto, é preciso reconhecer a necessidade de que os profissionais de saúde, em geral, estejam implicados a fim de que a população receba a assistência baseada em evidências científicas e que as terapêuticas estejam voltadas para redução de fissura e suporte para abstinência. Estudos científicos correlacionam as adipocinas como possíveis marcadores de doenças metabólicas, tais como obesidade, diabetes, doenças cardiovasculares e; alguns autores sugerem como possível marcador para dependência química, evidenciando a necessidade de novas pesquisas relacionadas ao tema.

Apesar de a literatura sugerir a leptina, adiponectina e a grelina como marcadores no tratamento de alcoolistas e tabagistas, a escassez de trabalhos ainda justifica pesquisas na área já que diversos fatores podem influenciar no estado de fissura e na manutenção da abstinência. Sendo assim, a relevância do presente estudo pauta-se no percentual elevado de tabagistas, na necessidade de intervenções eficazes para redução do consumo e na necessidade de reduzir a fissura e o desconforto na fase de abstinência, além de avaliar possíveis marcadores biológicos no acompanhamento do tratamento para a abstinência tabácica e a influência de fatores nessa situação-problema.

Pretende-se comprovar que as leptinas, adiponectinas e as grelinas podem ser consideradas como marcadores biológicos para o tratamento do tabagista podendo suas alterações influenciar na intensidade da fissura e conquista da abstinência. Confirmada a hipótese sustentada, tratamentos para tabagismo poderão pautar-se nos marcadores para fissura e abstinência contribuindo, significantemente, para a redução da fissura e manutenção da abstinência tabágica.

Traçou-se como objetivo geral analisar a associação entre os níveis séricos de leptina, adiponectina e grelina com a fissura e abstinência em tabagistas.

\section{METODOLOGIA}

Trata-se de uma pesquisa de natureza quantitativa do tipo intervenção clínica, realizada com amostra não probabilística tendo como campo o Centro Interdisciplinar de Pesquisa e Intervenção em Tabagismo do Hospital Universitário da Universidade Federal de Juiz de Fora (CIPIT/ HU-UFJF), no Estado de Minas Gerais, no Brasil, realizada no período de 2015 a 2018, durante o Programa de Pós-Graduação em Ciências Biomédicas da Universidade Federal Fluminense (UFF).

O trabalho realizado pelo CIPIT abrange atividades de prevenção, tratamento e controle do tabagismo, de abordagem multidisciplinar, com base nas diretrizes do Programa Nacional de Controle do Tabagismo (PNCT) do Instituto Nacional do Câncer (INCA). Para esta pesquisa, adotou-se como campo o Ambulatório de prevenção, tratamento e controle do tabagismo, integrado ao CIPIT, que funciona no primeiro andar do Hospital Universitário HU/UFJF, onde também se situa o Laboratório de Análises Clínicas onde foram coletadas as amostras de sangue para as dosagens séricas dos participantes. 
A amostra da pesquisa faz parte do banco de dados do grupo de pesquisa, que tem o total de 132 (cento e trinta e dois tabagistas), porém ao término dos quatro meses, apenas 29 (vinte e nove) concluíram o período proposto, sendo então a amostra final da pesquisa.

Compreende a participação de pessoas tabagistas de ambos os gêneros que buscam espontaneamente o CIPIT em busca de tratamento de cessação tabágica em regime ambulatorial, onde recebem intervenção intensiva em encontros semanais no primeiro mês e depois em dois encontros quinzenais e após, mensais até completarem doze meses de tratamento.

Como critérios de inclusão, adotou-se: a demanda espontânea para o grupo de cessação do tabagismo no campo escolhido, já com o diagnóstico médico de tabagismo de acordo com a Classificação Internacional das Doenças (CID-10), tendo como uso principal de droga o tabaco, maioridade de dezoito anos e, obviamente, tendo aceitado em participar da pesquisa, preenchendo o Termo de Consentimento Livre e Esclarecido (TCLE), conforme modelo preconizado pela legislação vigente.

Os critérios estabelecidos como exclusão foram estar gestante, possuir outras doenças crônicas, degenerativas, autoimunes ou comorbidade severa, não possuir capacidade cognitiva suficiente para participar da pesquisa e não ter score para dependência tabágica, de acordo com o protocolo Fagerstrom.

Para atingir os objetivos deste estudo, utilizou-se como instrumento para coleta de dados a ficha de anamnese clínica do Programa de Tabagismo, a qual contém o Teste de Dependência de Nicotina de Fagerstrom e o Questionário de avaliação da fissura Questionnaire of Smokimg UrgesBrief (QSU- Brief) - versão Brasil.

Os dados coletados foram armazenados no banco de dados do Grupo de Pesquisa do Tabagismo. A ficha de anamnese clínica do Programa do Tabagismo é composta por dez etapas, a saber: identificação do paciente; dados de encaminhamento; anamnese clínica; exame físico; exames complementares; história tabágica; história social e familiar; avaliação do grau de dependência (Fagerstrom), transtornos mentais e observações gerais. Para identificação do comportamento dos sujeitos participantes e o grau de fissura, foi utilizado como instrumento de coleta de dados o QSU- Brief.

No primeiro dia de acompanhamento, realizou-se a anamnese clínica dos participantes por profissional de saúde integrante da equipe do programa, devidamente capacitado para o tratamento do tabagismo. Em seguida, acompanhados por equipe multiprofissional do CIPIT-HU/UFJF, os participantes fizeram a avaliação nutricional antropométrica, sendo mensurados o peso corporal, altura, circunferência de cintura, circunferências de quadril e braquial e o índice de massa corporal (IMC), preenchendo ainda os questionários específicos.

Ainda no primeiro dia, os participantes coletaram sangue para avaliação clínica das condições endócrina e metabólica e para mensuração das dosagens séricas de adiponectina, leptina e grelina, realizados no Laboratório de Análises Clínicas do HU/UFJF. Os participantes mantiveram jejum de 12 horas para realização do exame, sendo coletado $10 \mathrm{ml}$ de sangue em veia periférica de membros superiores.

Para as dosagens da adiponectina, grelina e leptina, os soros foram armazenados estocados à temperatura de menos 80 graus centígrados até análise pelo método ELISA, utilizando kits específicos para humanos de cada uma das dosagens séricas, adquiridos pelo grupo de pesquisa. Orientações prévias quanto ao preparo, coleta e riscos referentes ao exame foram prestados pela equipe durante a reunião que antecedeu o primeiro encontro de intervenção aos participantes, bem como referente a toda pesquisa, estando ainda registrada no TCLE. Os exames de dosagens séricas foram repetidos quatro meses depois.

Após o primeiro dia de acompanhamento, os participantes deram início às quatro sessões estruturadas semanais para a abstinência tabágica com duração de um mês e, aqueles que permaneceram deram prosseguimento ao programa estabelecido. Após um mês, realizou-se nova avaliação antropométrica e depois ao término de quatro meses, sendo este o parâmetro utilizado nesta pesquisa, ou seja, mensuraçôes do início e quatro meses após.

Analisou-se a fissura pelo QSU-Brief semanalmente e, para confirmar a abstinência e o valor de monóxido de carbono expirado (COex), foi mensurado no início e após mensalmente durante o tratamento, até o quarto mês. Para este procedimento foi usado o aparelho monoxímetro de carbono (Pico Plus - piCO+Smokerlyzer), adquirido para pesquisas do grupo de pesquisa. $\mathrm{O}$ valor de COex em ppm foi classificado em: não fumante (0 a 3 ppm), fumante leve ( 4 a 6 ppm) e fumante pesado (7 ppm ou mais).

Neste estudo, realizou-se a análise estatística descritiva. Os dados inicialmente foram analisados quanto à normalidade usando o Teste para uma amostra Kolmogorov-Smirnov. Comparações de variáveis contínuas foram conduzidas por testes não paramétricos. Para análise de variância, adotou-se como critério o indicado para amostras independentes, sendo utilizado o teste $\mathrm{t}$ simples Student para comparar variáveis categóricas iniciais e 4 meses depois.

Foi usado o software Statistical Package for the Social Sciences (SPSS) 23.0. O tratamento de análise de dados se deu por análise dos resultados das testagens pelo software estatístico, sendo analisada a concentração de adiponectina, grelina e leptina correlacionando as dosagens no período inicial de tratamento e quatro meses após e o desfecho quanto ao status tabágico, correlacionando ainda ao Questionário de Fissura do tabagismo naqueles que permaneceram até o quarto mês de tratamento.

Em todas as análises, para efeito de interpretação, o limite de erro tipo I foi de até $5 \%(p<0,05)$. Pressupostos dos testes foram respeitados. Avaliações de associações foram feitas por testes de correlações entre adipocinas e QSU- Bref. A leptina foi ajustada pelo IMC e pela circunferência de cintura.

Neste estudo foram respeitados os princípios éticos da realização de pesquisa com seres humanos determinados pela Resolução n. ${ }^{\circ}$ 466/12 do Conselho Nacional de Saúde (CNS). Os dados coletados e os achados foram utilizados para análise, in- 
terpretação, e divulgação de informações em saúde por meio de artigos e outras publicações científicas, garantindo o sigilo dos participantes. A amostra faz parte do banco de dados do grupo de pesquisa, aprovada pelo Comité de Ética e Pesquisa (CEP) da UFJF (CAAE: 0067.0180.42011/Parecer n. $\left.{ }^{\circ} 081 / 2011\right)$.

A autorização individual para a coleta de dados deu-se formalmente por escrito com o preenchimento do TCLE após autorização do CEP e, posteriormente, foram entregues aos sujeitos participantes do estudo, para que tomassem ciência e registrassem o consentimento, durante a reunião com a equipe a qual antecedeu o primeiro encontro do grupo.

\section{RESULTADOS E DISCUSSÃO}

Como limitações do estudo, podemos considerar o reduzido tamanho da amostra final em função do elevado número de pessoas que não completaram o tratamento para cessação tabágica, a perda de registro no banco de dados de algumas dosagens séricas e dados antropométricos, a falta de investigação quanto à eficácia da reposição de nicotina e da terapia medica- mentosa e a impossibilidade de avaliar os que deixaram o tratamento, que podem ter tido fissura maior e, por isso, abandonaram o tratamento, não conseguindo manter-se abstênicos. A amostra inicial foi de 132 participantes e apenas 29 concluíram os quatro meses.

No que se refere à amostra inicial, considerando a média e desvio padrão de $49,7 \pm 10,8$ ( $\mathrm{n}=132)$, a idade mínima foi de 20 anos e a máxima de 77 anos. Podemos dizer que a faixa etária variou entre adultos jovens a idosos. Percebe-se que a média de idades tem equivalência com os dados do INCA, nos quais a média dos fumantes no que se refere à faixa etária de maior consumo é entre 45 e $54 \operatorname{anos}^{(19)}$. Considera-se também o percentual de alto índice de óbitos decorrente do tabagismo, o que pode caracterizar menor percentual em idosos e a diminuição do uso em jovens na atualidade em decorrência nas medidas adotadas pelo Brasil para redução de consumo e ao controle de venda de cigarros à menores de idade.

No que se refere à amostra final, considerando a média e desvio padrão de $50,7 \pm 10,5$ $(\mathrm{n}=29)$, a idade mínima foi de 30 anos e a máxima de 72 anos. Contatou-se a desis- tência dos adultos jovens e dos idosos acima de 72 anos. Em geral os tabagistas adultos jovens não apresentam comorbidades severas e, por isso, podem não aderir ao tratamento por não se dar conta da gravidade do tabagismo. À população mais velha, se considerarmos a média da idade inicial de consumo do tabaco, possuem maior tempo de dependência, tendo maior dificuldade para manter-se abstênicos.

A amostra inicial foi de 87 mulheres e 44 homens, tendo 1 item sobre sexo não declarado durante a coleta de dados. O maior número ser de mulheres pode ocorrer em virtude de elas buscarem mais os serviços de saúde para tratamento do que os homens, já que no Brasil, o tabagismo ainda é maior entre os homens $(12,8 \%)$ do que entre as mulheres $-9 \%{ }^{(20)}$.

A amostra final foi composta por $22 \mathrm{mu}-$ lheres e 7 homens. A redução da amostra indica pouca adesão e dificuldade em permanecer no tratamento. Esse dado encontra-se equivalência na literatura já que, em grau de dependência alto, a chance de manter-se abstêmio é muito baixa. Considera-se que a partir da soma de seis pontos no resultado do teste de dependência tabágica que a pessoa terá dificuldade de parar de fumar ${ }^{(21)}$.

Tabela 1. Parâmetros antropométricos no início e ao término do tratamento e o estado tabágico dos voluntários (média e desvio padrão). Juiz de Fora, MG, Brasil, 2015-2018

\section{PARÂMETROS DE CONTROLE}

Peso corporal

Índice de massa corporal

Circunferência de cintura

Circunferência abdominal
AMOSTRA

Fumantes

Pararam de fumar

Fumantes

Pararam de fumar

Fumantes

Pararam de fumar

Fumantes

Pararam de fumar

\section{INÍCIO DO TRATAMENTO}

$$
\mathrm{n}=07
$$

$\mathrm{n}=21$

$\mathrm{n}=07$

$\mathrm{n}=21$

$\mathrm{n}=07$

$\mathrm{n}=21$

$\mathrm{n}=07$

$\mathrm{n}=21$
$71,1 \pm 15,0$

$70,8 \pm 15,1$

$29,1 \pm 5,6$

$27,8 \pm 5,7$

$88,0 \pm 13,1$

$87,8 \pm 13,2$

$94,6 \pm 15,3$

$94,9 \pm 13,6$

\section{TÉRMINO DE} TRATAMENTO
Tabela 2. Níveis séricos de leptina em voluntários que finalizaram o tratamento ajustados para IMC e o estado tabágico final. Juiz de Fora, MG, Brasil, 2015-2018

$$
\text { ESTADO TABÁGICO }
$$

Fumantes

INÍCIO DO
TRATAMENTO

$14,1 \pm 6,7(n=7)$

$12,5 \pm 6,8(n=22)$
TÉRMINO DO TRATAMENTO

$14,9 \pm 7,9(n=7)$

$13,5 \pm 6,1(n=21)$
Analisando os dados da tabela acima, constatamos que não houve significância dos dados antropométricos iniciais e finais entre tabagistas e não tabagistas durante os quatro meses de tratamento, embora a literatura registre o aumento das medidas com a cessação. 


\section{artigo}

Paiva, S.S.; Aguiar, A.S.; Silva, L.P.G.O.; Amorim, G.P.; Avellar, B.; Fonseca, V.A.S.;

Niveis séricos de adipocinas como preditores no sucesso do tratamento de tabagistas

Conforme a tabela acima, observou-se que os níveis de leptina, quando comparados entre o início e o quarto mês do tratamento entre tabagistas e abstêmios, não sofreram diferenças significativas ao nível de $\mathrm{p}<0,05$ na comparação entre grupos. Foram realizados teste $\mathrm{t}$ de Student para comparação entre a leptina inicial e final após teste de normalidade, estando em consonância com estudo ${ }^{(22)}$, cujos autores também não encontraram correlação entre fumantes crônicos e níveis de leptina sérica.

Ao realizar as testagens, encontramos significância apenas naqueles que pararam de fumar, ou seja, a leptina aumenta com a redução da fissura, diferente do achado de autores ${ }^{(10)}$, em que maiores concentrações de leptina foram associadas ao maior desejo e dificuldade em alcançar a abstinência, porém encontrou concordância com os achados no estu$\mathrm{do}^{(23)}$, onde a análise indicou que aumento de leptina no início da cessação prediz abstinência. Sendo assim, podemos considerar o aumento das dosagens de leptina ajustada para circunferência de cintura como marcador para abstinência.

De acordo com os dados acima, aqueles que pararam de fumar ao término tinham nível elevado no início de adiponectina se comparados com aqueles que permaneceram fumantes, podendo então ser considerada a adiponectina como preditor para a cessação tabágica.

Tabela 3. Níveis séricos de leptina em voluntários que finalizaram o tratamento ajustados para circunferência de cintura e o estado tabágico final. Juiz de Fora, MG, Brasil, 2015-2018

\section{ESTADO TABÁGICO}

Fumantes

Pararam de fumar

Tabela 4. Níveis séricos de adiponectina em voluntários que finalizaram o tratamento e o estado tabágico final. Juiz de Fora, MG, Brasil, 2015-2018

\section{INÍCIO DO} TRATAMENTO

$12,9 \pm 5,2(n=7)$

$12,8 \pm 5,3(n=22)$

\section{TÉRMINO DO TRATAMENTO}

$16,3 \pm 6,4(n=7)$ $16,3 \pm 5,2(n=22)$

\section{ESTADO TABÁGICO}

Fumantes

Pararam de fumar
Tabela 5. Níveis séricos de grelina em voluntários que finalizaram o tratamento e estado tabágico final. Juiz de Fora, MG, Brasil, 2015-2018

\section{ESTADO TABÁGICO}

Fumantes

Não fumantes

Tabela 6. Média e desvio padrão dos Scores do Teste de Avaliação da fissura QSU Inicial e Final dos voluntários que concluíram o tratamento e o estado tabágico final. Juiz de Fora, MG, Brasil, 2015-2018

\section{ESTADO TABÁGICO}

Fumantes
Pararam de fumar

\section{INÍCIO DO} TRATAMENTO

$(n=7) 27,1 \pm 26,4$

$(n=17) 12,5 \pm 19,0$
TÉRMINO DO TRATAMENTO

(n= 7) $6,7 \pm 3,4$
Para autores ${ }^{(24)}$, as alterações dos níveis plasmáticos de adiponectina após a cessação do tabagismo comparando às dosagens iniciais e até o terceiro mês de tratamento, no grupo que parou de fumar, aumentou significativamente após 4 semanas, porém não mencionou se a mesma já estava elevada no início, se comparada com a concentração normal.

Após análise estatística, não houve significância, provavelmente pelo desvio padrão alto, assim como também ocorreu no estudo ${ }^{(25)}$, onde não foram encontradas diferenças em relação aos níveis de grelina entre fumantes e não fumantes.

De acordo com a tabela, todos os participantes da pesquisa na fase inicial tinham score de fissura moderada. Ao término, aqueles que permaneceram tabagistas reduziram para o score de fissura leve. Já aqueles que conseguiram deixar de fumar, reduziram para fissura mínima. Destacamos que em todos os participantes houve redução de danos, já que no início apresentavam fissura intensa.

\section{CONCLUSÃO}

Foi analisada a associação dos níveis séricos de leptina, adiponectina e grelina com a fissura e abstinência em tabagistas, comparando os dados do início e após quatro meses de tratamento, analisando a influência na intensidade da fissura e na manutenção da abstinência, correlacionando ainda com os dados revelados no QSU daqueles que permaneceram até o quarto mês de tratamento.

$\mathrm{Na}$ análise dos dados antropométricos não houve significância assim como nos níveis séricos de leptina ajustada para IMC entre aqueles que pararam de fumar e os que permaneceram fumantes.

Em relação à leptina ajustada pela circunferência de cintura ao realizar a testagem, encontramos significância naqueles que pararam de fumar, ou seja, a leptina aumenta com a redução da fissura, podendo ser considerado como marcador para fissura e risco de recaída.

Quanto às adiponectinas, aqueles que pararam de fumar ao término tinham 
nível elevado, quando comparadas às dosagens iniciais dos que permaneceram fumantes, podendo ser considerada como preditor para a cessação tabágica.

Quanto às análises dos níveis séricos de grelina, não mostraram significância entre aqueles que pararam de fumar e os que permaneceram fumantes.

Com relação ao grau da intensidade da fissura, todos os participantes possu- íam score do QSU de fissura moderada no início do tratamento e ao término dos 4 meses; os que permaneceram fumantes apresentavam fissura leve e os que pararam de fumar, fissura mínima.

Constatamos que houve redução de danos em todos os participantes em virtude da cessação do tabagismo em 22 deles e da redução do grau da fissura inicial para o final nos 7 que permaneceram tabagistas.
Concluímos que as adipocinas podem ser consideradas não somente marcadores para fissura e risco de recaída, mas também preditores para o sucesso do tratamento de tabagistas. Esta pesquisa, por ter identificado a leptina como marcador para fissura e risco de recaída e ainda a adiponectina como marcador e preditor para cessação tabágica, contribuirá significativamente para o sucesso do tratamento de tabagistas.

\section{REFERÊNCIAS}

1. Aguiar-Nemer AS, et al. Leptin influence in craving and relapse of alcoholics and smokers. Journal of clinical medicine research. 2013; 5(3): 164.

2. Organização Mundial da Saúde. MPOWER: um plano de medidas para reverter a epidemia de tabagismo. OMS, 2008.

3. Organização Mundial da Saúde. Classificação Internacional de Doenças e Problemas Relacionados à Saúde - Décima Revisão- (CID 10). 4. ed. São Paulo: Editora da Universidade de São Paulo; 1997.

4. American Psychiatric Association. Manual Diagnóstico e estatística de doenças mentais/DSM V. 5. ed. Rio de Janeiro: Artmed; 2014.

5. Ministério da Saúde, Instituto Nacional do Câncer (BR). Convenção - Quadro para o controle do Tabaco. Rio de Janeiro, 2011.

6. Centro Brasileiro de Informação Sobre Drogas Psicoterápicas. Departamento de psicobiologia da Unifesp UFSP. Escola Paulista de Medicina. Drogas Psicotrópicas. São Paulo: FAPESP.

7. Pierce RC, Kumaresan V. The mesolimbic dopamine system: the final common pathway for the reinforcing effect of drugs of abuse? Neuroscience \& biobehavioral reviews. 2006; 30(2):215-238.

8. Volkow ND, Wise RA. How can drug addiction help us understand obesity? Nature neuroscience. 2005; 8(5):555.

9. Araujo RB, et al. Craving e dependência química: conceito, avaliação e tratamento. J bras psiquiatr. 2008; 57(1):57-63.

10. Gomes AS, et al. Influence of the leptin and cortisol levels on craving and smoking cessation. Psychiatry research. 2015; 229(1-2):126-132.

11. Araujo RB, et al. Validation of the Brazilian version of Questionnaire of Smoking Urges-Brief. Archives of Clinical Psychiatry. 2007; 34(4):166-175.

12. Al'Absi M, et al. Circulating leptin levels are associated with increased craving to smoke in abstinent smokers. Pharmacology Biochemistry and Behavior. 2011; 97(3):509-513.

13. Reseland JE, et al. Cigarette smoking may reduce plasma leptin concentration via catecholamines. Prostaglandins, leukotrienes and essential fatty acids. 2005; 73(1):43-49.

14. Roubos EW, Dahmen M. Leptin and the hypothalamo-pitui- tary-adrenal stress axis. Gen. Comp. Endocrinol. 2012; 177:2836.

15. Kubota N, et al. Adiponectin stimulates AMP-activated protein kinase in the hypothalamus and increases food intake. Cell metabolism. 2007; 6(1):55-68.

16. Kumsar NA, Dilbaz N. Relationship between craving and ghrelin, adiponectin, and resistin levels in patients with alcoholism. Alcoholism: Clinical and Experimental Research. 2015; 39(4):702-709.

17. Lee $H$, et al. Increased leptin and decreased ghrelin level after smoking cessation. Neuroscience letters. 2006; 409(1):4751.

18. Inoue $K$, et al. Early effects of smoking cessation and weight gain on plasma adiponectin levels and insulin resistance. Internal Medicine. 2011; 50(7):707-712.

19. Instituto Nacional do Câncer (BR). Manual. Plano de Implantação da Abordagem e Tratamento do Tabagismo na Rede SUS, 2011.

20. Ministério da Saúde, Secretaria de Atenção à Saúde, Departamento de Atenção Básica (BR). Estratégias para o cuidado da pessoa com doença crônica: o cuidado da pessoa tabagista/ Ministério da Saúde, Secretaria de Atenção à Saúde, Departamento de Atenção Básica. - Brasília: Ministério da Saúde, 2015.154 p.

21. Halty LS, et al. Pesquisa sobre tabagismo entre médicos de Rio Grande, RS: prevalência e perfil do fumante. J Pneumol. 2002; 28(2).

22. Neslihan AK, Dilbaz N. Relationship between craving and ghrelin, adiponectin, and resistin levels in patients with alcoholism. Alcoholism: Clinical and Experimental Research. 2015; 39(4):702-709.

23. Lemieux $A$, et al. Changes in circulating leptin levels during the initial stage of cessation are associated with smoking relapse. Psychopharmacology. 2015; 232(18):3355-3361.

24. Won WY, et al. Changes of plasma adiponectin levels after smoking cessation. Psychiatry investigation. 2014 Apr; 11(2):173-8.

25. Komiyama M, et al. Analysis of changes on adiponectin levels and abdominal obesity after smoking cessation. PloS one. 2018; 13(8):e0201244. 